\title{
Escala diagramática para avaliação da severidade do oídio em castanhas de caju
}

\author{
Joilson Silva Lima ${ }^{1}$, Marlon Vagner Valentim Martins ${ }^{2}$, Francisco Marto Pinto Viana ${ }^{2}$, José Emilson Cardoso²
}

${ }^{1}$ Departamento de Fitotecnia, Universidade Federal do Ceará, 60021-970, Fortaleza-Brasil; ${ }^{2}$ Laboratório de Fitopatologia, Embrapa Agroindústria Tropical, 60511-110, Fortaleza-Brasil.

Autor para correspondencia: Marlon Vagner Valentim Martins (marlon.valentim@embrapa.br)

Data de chegada: 13/04/2017. Aceito para publicação em: 28/09/2017.

$10.1590 / 0100-5405 / 178543$

\section{RESUMO}

Lima, J.S.; Martins, M.V.V; Viana, F.M.P; Cardoso, J.E. Escala diagramática para avaliação da severidade do oídio em castanhas de caju. Summa Phytopathologica, v.44, n.3, p.252-260, 2018.

O oídio, causado pelo fungo Pseudoidium anacardii é atualmente a principal doença do cajueiro (Anacardium occidentale). Devido à importância dessa fitomoléstia e à ausência de métodos padronizados para a sua quantificação, uma escala diagramática descritiva com os níveis 0 (Nota 0), 5 (Nota 1), 17,5 (Nota 2), 37,5 (Nota 3), 62,5 (Nota 4) e 87,5\% de área superficial lesionada (Nota 5) foi proposta, elaborada e testada para a acurácia, precisão e reprodutibilidade das estimativas de severidade do ataque do fungo em castanhas de cajueiro. A escala foi testada por vinte pessoas (dez com experiência e dez sem experiência) que utilizaram cinquenta castanhas com diferentes níveis de severidade, previamente mensuradas com auxílio do programa Assess ${ }^{\circledR}$, para serem avaliadas sem e com o uso da escala diagramática descritiva proposta. Regressões lineares simples entre a severidade real (mensurada eletronicamente) e a severidade estimada por cada avaliador foram utilizadas para a determinação da acurácia e precisão. A reprodutibilidade foi obtida por regressões lineares entre as severidades estimadas da mesma unidade amostral por diferentes avaliadores, combinados em pares, com e sem o uso da escala. O emprego da escala diagramática descritiva para avaliação da severidade do oídio em castanhas de cajueiro mostrou ser de fácil utilização e melhorou os níveis de acurácia, precisão e reprodutibilidade das estimativas, independente da experiência do avaliador. Com o seu uso em levantamento de campo, estudos epidemiológicos, resistência e estudos de medidas de controle do oídio, será possível obter informações mais precisas sobre a intensidade da doença nesse importante patossistema, podendo ser usada por estudantes, técnicos, pesquisadores e produtores para quantificar sintomas do oídio em plantas de cajueiro.

Palavras-chave: Anacardium occidentale, Pseudoidium anacardii, Acurácia, Precisão, Reprodutibilidade.

\section{ABSTRACT}

Lima, J.S.; Martins, M.V.V; Viana, F.M.P; Cardoso, J.E. Diagrammatic scale for powdery mildew severity evaluation in cashew nuts. Summa Phytopathologica, v.44, n.3, p.252-260, 2018.

Powdery mildew, caused by the fungus Pseudoidium anacardii, is currently the main disease of cashew tree (Anacardium occidentale). In view of the importance of such plant disease and the absence of standardized methods for its quantification, a descriptive diagrammatic scale was proposed with levels 0 (Score 0), 5 (Score 1), 17.5 (Score 2), 37.5 (Score 3), 62.5 (Score 4) and $87.5 \%$ injured superficial area (Score 5), and was elaborated and tested for accuracy, precision and reproducibility of severity estimates of fungal attack on cashew nuts. The scale was tested by twenty people (ten experienced and ten inexperienced people) who used fifty nuts showing different severity levels, previously measured with the Assess ${ }^{\circledR}$ program, to be evaluated without and with the proposed descriptive diagrammatic scale. Simple linear regressions between the actual severity (measured electronically) and the severity estimated by each evaluator were used to determine accuracy and precision. Reproducibility was obtained based on linear regressions between severities of the same sampling unit estimated by different raters, combined in pairs, with and without the scale. The descriptive diagrammatic scale to assess powdery mildew severity in cashew nuts was easy to use and improved the levels of accuracy, precision and reproducibility of the estimates, regardless of the evaluator's experience. Using the scale in field surveys and epidemiological, resistance and powdery mildew control measures studies will provide more precise information on the disease intensity in such an important pathosystem, besides allowing its use by students, technicians, researchers and farmers to quantify powdery mildew symptoms in cashew plants.

Keywords: Anacardium occidentale, Pseudoidium anacardii, accuracy, precision, reproducibility.

O cajueiro (Anacardium occidentale L.) é uma das mais importantes frutíferas cultivadas nas regiões semiáridas de clima tropical do mundo (34). O Brasil é atualmente um dos principais produtores de caju (10), sendo a região Nordeste responsável por mais de $98 \%$ da produção nacional, com destaque para o estado do Ceará como o maior produtor do país (17).

Apesar da rusticidade associada ao cajueiro, considerada uma planta bem adaptada às condições de regiões semiáridas, esta anacardiácea pode ser acometida por diversos problemas fitossanitários (12), principalmente ocasionados por fungos, os quais comprometem sobremaneira o seu desenvolvimento.

Atualmente, o fungo Pseudoidium anacardii (F. Noack) U. Braun \& R. T. A. Cook, causador do oídio do cajueiro, é o principal patógeno associado a essa cultura. Descrito pela primeira vez associado a plantas de cajueiro por Noack, em 1898 (12), trata-se de um fungo Ascomiceto da ordem Erisyphales (4), responsável por atacar diversas espécies de plantas (5), notadamente anacardiáceas (36).

Em plantas de cajueiro, o oídio é responsável por ocasionar sintomas severos em folhas, inflorescências, e frutos (6), apresentando-se como uma séria ameaça à cultura (36). Devido a sua rápida disseminação e 
agressividade (15), na ausência de monitoramento e adoção de medidas de controle, o oídio do cajueiro pode ocasionar sérios danos (21).

Dependendo das condições climáticas, o fungo infecta inicialmente os tecidos jovens, provocando um revestimento pulverulento de coloração branco-acinzentado na superfície dos órgãos afetados, assemelhando-se à cinza vegetal (6), ocasionado pelo crescimento micelial e formação de conídios do fungo. As folhas e brotações ficam malformadas e descoloridas. Os frutos e pedúnculos também se tornam descoloridos, racham e atrofiam.

Apesar da importância do oídio em cajueiro, inexistem métodos padronizados e de fácil utilização para a quantificação da severidade dessa enfermidade, o que segundo Barguil et al. (3), é fator limitante em estudos epidemiológicos e de comparação de medidas de controle. A severidade do oídio em cajueiro tem sido estimada, principalmente utilizando-se escalas descritivas de sintomas $(7,6)$.

Um sistema de análise da intensidade da fitomoléstia que apresente elevada acurácia, precisão e reprodutibilidade é essencial para qualquer programa de manejo de doenças de plantas bem sucedido $(28,31)$, servindo de guia para o avaliador (1). Além disso, o sistema necessita ser de fácil e rápida utilização para uma ampla gama de condições (26), e deve apresentar níveis suficientes que representem todos os estádios de desenvolvimento da doença (2).

Escalas diagramáticas são representações ilustradas de uma série de plantas ou partes dela como folhas, flores ou frutos, apresentando sintomas em diferentes níveis de ataque por patógenos $(1,29)$. Essas escalas constituem-se atualmente na principal ferramenta de avaliação da severidade para muitas doenças $(2,3,8,9,11,13,14,19,20,22$, $28,31,32,39,40$ ), podendo ser utilizadas na quantificação dos danos, resistência varietal e em experimentos de controle da doença $(9,16)$. O uso de uma escala diagramática descritiva constitui-se, portanto, numa excelente alternativa para a avaliação do oídio do cajueiro.

Na elaboração de escalas, a acurácia está relacionada à proximidade de uma estimativa visual a um valor real de quantidade da doença avaliada. A precisão refere-se à variação ou repetibilidade associadas com uma estimativa. Já a reprodutibilidade representa a ausência de variação em estimativas quando a mesma amostra de doença é avaliada por diferentes avaliadores $(3,26,27,33)$.

Para verificar a qualidade das estimativas propiciadas pela escala diagramática, os níveis de acurácia, precisão e reprodutibilidade devem ser analisados $(26,33)$. Além disso, as subdivisões de uma boa escala deve respeitar as limitações da acuidade visual humana, definidas pela lei de estímulo-resposta de Weber-Fechner, na qual a acuidade visual é proporcional ao logaritmo da intensidade do estímulo $(16,31)$.

A estimativa da real proporção ou percentagem da área lesionada é o motivo pelo qual se desenvolvem métodos de quantificação de doenças, pois dessa forma previnem-se sérios erros na aquisição de dados e a subjetividade das estimativas visuais é minimizada $(2,3)$.

Nesse contexto, devido à ausência de métodos padronizados para a quantificação da intensidade do oídio do cajueiro, objetivou-se com este trabalho elaborar e validar uma escala diagramática descritiva para a avaliação da severidade do oídio em castanhas de caju e analisar a qualidade dos níveis de acurácia, precisão e reprodutibilidade das estimativas geradas com sua utilização.

\section{MATERIAL E MÉTODOS}

Para a elaboração da escala diagramática descritiva foram coletadas 100 castanhas do clone de cajueiro anão BRS 189, com diferentes níveis de severidade do ataque do oídio, em uma área do Campo Experimental da Embrapa Agroindústria Tropical (4 ${ }^{\circ} 11$, $16^{\prime}$ 'S; $38^{\circ} 29^{\prime} 50^{\prime}$ ' W, $77 \mathrm{~m}$ ), localizado no município de Pacajus, no estado do Ceará. Cada castanha teve suas duas faces fotografadas com uma câmara digital (Sony ${ }^{\circledR}$, modelo DSC-W530), com resolução de 300 dpi. As imagens de cada castanha foram analisadas em computador quanto ao percentual de área lesionada, utilizando o programa Assess ${ }^{\circledR}$ (18), obtendo-se a severidade real do oídio em termos percentuais.

Baseando-se na lei de Weber-Fechner de acuidade visual (16), bem como na forma, distribuição e frequência das lesões nas castanhas, foi confeccionada uma escala diagramática descritiva com seis níveis de severidade. Na validação da escala diagramática descritiva proposta foram utilizadas 50 castanhas entre aquelas coletadas inicialmente $(11,28,39,40)$, com diferentes níveis de intensidade da doença. A severidade foi avaliada por vinte pessoas, sendo dez com experiência nesse tipo de análise (A, B, C, D, E, F, G, H, I e J) e dez sem experiência (K, L, M, N, O, P, Q, R, S e T). Inicialmente, os avaliadores estimaram a severidade do oídio em castanhas de cajueiro sem o uso da escala diagramática descritiva proposta. Na segunda etapa da validação, eles repetiram a avaliação com o auxílio da escala.

A partir dos dados de cada avaliador, determinou-se a acurácia e a precisão por meio da regressão linear simples entre a severidade real estimada eletronicamente pelo programa Assess ${ }^{\circledR}$ (variável independente) e a severidade estimada visualmente pelo avaliador (variável dependente), sem e com o uso da escala.

A acurácia, que representa o grau de proximidade entre a estimativa média e a severidade real de doença (23), foi determinada por meio dos parâmetros " $a$ " (intercepto da regressão linear) e " $b$ " (coeficiente angular da reta) da equação de regressão linear, comparando-os, respectivamente, aos valores de $0 \mathrm{e} 1$, pelo teste $t$ $(P<0,05 \%)$, sem e com o uso da escala $(13,14,22,40)$. Valores de intercepto $(a)$ significativamente diferentes de 0 (zero) sugerem a presença de desvios constantes, indicando superestimativa $(>0)$ ou subestimativa $(<0)$ da severidade real a níveis baixos de intensidade do oídio nas castanhas; enquanto valores de coeficiente angular da reta (b) que diferem significativamente de 1 (um) implicam na existência de desvios sistemáticos, indicando superestimativa $(>1)$ ou subestimativa $(<1)$ da severidade real em todos os níveis de intensidade do oídio $(2,11,27,32,33)$.

A precisão, que se refere à variação associada com a estimativa da quantidade de doença (1), foi obtida pelo coeficiente de determinação $\left(R^{2}\right)$ da regressão linear e pela variação dos erros absolutos, expressa pela diferença entre o valor da severidade do oídio estimada e o valor real, para cada avaliador sem e com o uso da escala, considerando que quanto mais próximo de 1 (um) fosse o coeficiente de determinação $\left(R^{2}\right)$, maior seria a precisão $(2,9$, $11,13,20,28,40)$.

A reprodutibilidade das estimativas da escala, que também pode ser utilizada como um indicativo de precisão de um método de avaliação de doenças $(30,33)$, foi determinada pelos valores dos coeficientes de determinação $\left(R^{2}\right)$ obtidos das regressões lineares entre as severidades estimadas da mesma unidade amostral por diferentes avaliadores, combinados em pares, com e sem o uso da escala diagramática descritiva proposta $(24,26,33)$, verificandose a reprodutibilidade dentro do grupo de avaliadores experientes, dentro do grupo dos inexperientes e dentro de um terceiro grupo, este composto por avaliadores experientes e inexperientes. 


\section{RESULTADOS E DISCUSSÃO}

A escala diagramática descritiva para a avaliação do oídio em castanhas de cajueiro foi elaborada com seis níveis de severidade da doença: 0 (Nota 0 = ausência de lesão na superfície da castanha), 5 (Nota $1=$ até $10 \%$ da área da superfície lesionada), 17,5 (Nota $2=$ entre 10 e $25 \%$ da área da superfície lesionada), 37,5 (Nota $3=$ entre 25 e $50 \%$ da área da superfície lesionada), 62,5 (Nota $4=$ entre 50 e $75 \%$ da área da superfície lesionada) e 87,5\% de área superficial lesionada (Nota 5 = acima de $75 \%$ da área da superfície lesionada), com sintomas típicos do ataque do fungo (Figura 1). Em virtude do grande numero de imagens coletadas e da heterogeneidade da severidade do oídio, foi possível localizar castanhas com níveis idênticos aos definidos pela escala. Assim, os desenhos apresentados, com lesões de formatos variados, tratam-se da reprodução de imagens reais e estão distribuídos de maneira a retratar os sintomas observados no campo.

A escala elaborada apresentou limite superior de severidade elevado, com o valor de $87,5 \%$, mas que assemelha-se ao estabelecido na elaboração da escala diagramática para avaliar a severidade do míldio do meloeiro (28), da ferrugem da soja (13), da mancha de Cylindrocladium em eucalipto (9), da brusone em espigas de trigo (22) e da antracnose do maracujá amarelo (11), fazendo-se necessário em virtude da alta frequência de castanhas com elevado percentual da superfície lesionada pelo oídio, com até $100 \%$ da área superficial atacada. Entretanto, limite superior de severidade com valores reduzidos foram colocados na confecção da escala da mancha marrom da alternaria de citros (37), do carvão da folha do caupi (26), da manchade-micosferela em morangueiro (25), da mancha-parda em arroz (20) e da pinta preta em frutos de mamoeiro (40), com percentuais de área lesionada que variaram entre 20 e $45 \%$, devido, principalmente à ausência de maiores intensidades de doença nos órgãos avaliados em condições de campo, levada muitas vezes pela queda precoce em função do ataque do patógeno.

A avaliação da severidade do oídio em castanhas de cajueiro com o uso da escala diagramática descritiva melhorou a acurácia e a precisão das estimativas para a maioria dos avaliadores, demonstrando que a mesma foi efetiva para a quantificação da doença.

Sem a utilização da escala, os valores do intercepto $(a)$ relacionados a $60 \%$ dos avaliadores experientes (B, D, E, G, I e J) e $70 \%$ dos inexperientes ( $\mathrm{L}, \mathrm{N}, \mathrm{O}, \mathrm{P}, \mathrm{R}, \mathrm{S}$ e $\mathrm{T}$ ) diferiram significativamente de zero $(P<0,05)$, indicando a presença de desvios constantes (Tabela 1$)$. A maioria dos avaliadores experientes e todos os avaliadores inexperientes superestimou consistentemente $(P<0,05)$ a severidade do oídio em castanhas de cajueiro sem o uso da escala (Tabela1), o que foi apontado pela presença de desvios constantes e positivos $(11,27)$ para todos os níveis de severidade da doença (Figura 2).

Com a utilização da escala, os valores do intercepto $(a)$ que diferiram significativamente de 0 (zero) $(P<0,05)$ foram de apenas $40 \%$ e $50 \%$, para os avaliadores experientes e inexperientes, respectivamente, mostrando uma significativa melhora nas estimativas da severidade da doença, com expressiva redução nos desvios (Tabela 1), indicando também uma discreta tendência de subestimativa por parte de alguns avaliadores (Figura 2Ac, 2Cc, 2Fc, 2Hc, 2Nc, 2Qc e 2Rc).

A superestimativa dos níveis de severidade das doenças, como o verificado para o oídio em castanhas de cajueiro, é comum em vários patossistemas quando as avaliações são realizadas sem o uso de escalas diagramáticas $(27,30,39)$, podendo ser reduzida com o uso da escala (11). A tendência dos avaliadores em subestimar os níveis de severidade do oídio em castanhas de cajueiro quando utilizaram a escala diagramática descritiva assemelha-se ao observado por outros autores na validação das escalas para a cercosporiose da alface (14), o míldio do meloeiro (28) e a pinta-preta em frutos de mamoeiro (40).

Sem o uso da escala diagramática, $50 \%$ dos valores do coeficiente angular da reta $(b)$ dos avaliadores experientes, e $60 \%$ dos valores dos inexperientes diferiram significativamente de 1 (um) $(P<0,05)$. Utilizando a escala, apenas $20 \%$ dos valores do coeficiente angular dos avaliadores experientes e $30 \%$ dos valores dos inexperientes diferiram de 1 (um) (Tabela 1), indicando que o uso da escala interferiu na

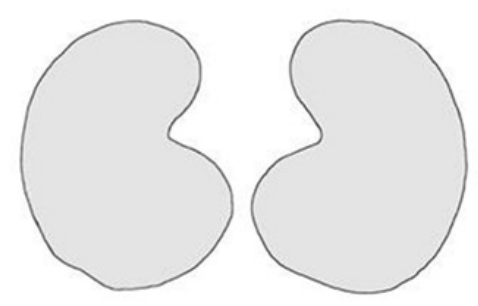

$\mathbf{0} \%$

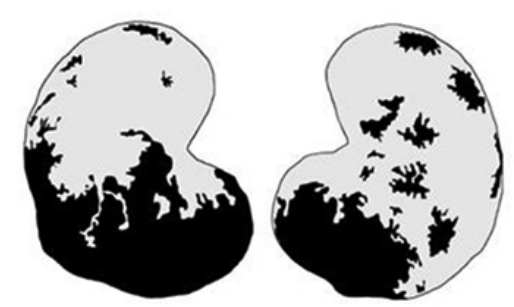

$37,5 \%$

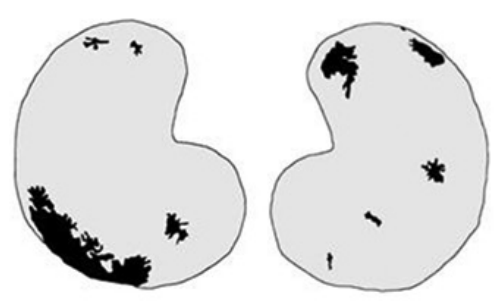

$\mathbf{5 \%}$

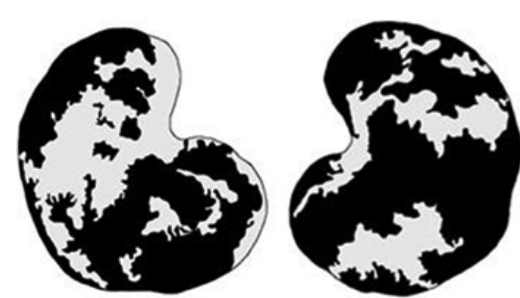

$62,5 \%$
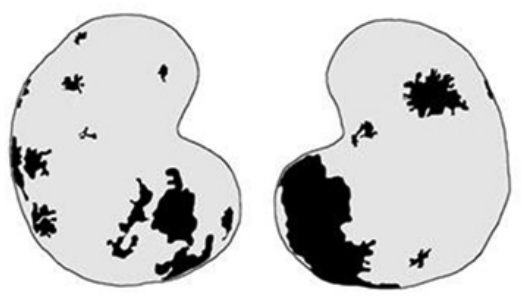

$17,5 \%$

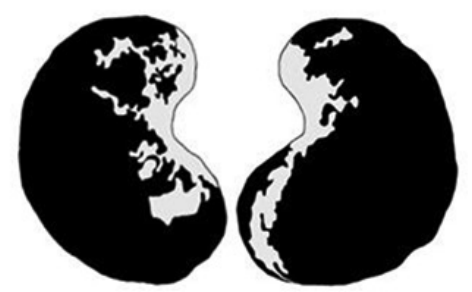

$87,5 \%$

Figura 1. Escala diagramática descritiva para a avaliação do oídio (Pseudoidium anacardii) em castanhas de cajueiro (Anacardium occidentale), indicando os seis níveis de severidade: $0 \%=$ Nota 0 (ausência de lesão na superfície da castanha); $5 \%=$ Nota 1 (até $10 \%$ da área da superfície lesionada); $17,5 \%=$ Nota 2 (entre 10 e $25 \%$ da área da superfície lesionada); $37,5 \%=$ Nota 3 (entre 25 e $50 \%$ da área da superfície lesionada); $62,5 \%=$ Nota 4 (entre 50 e $75 \%$ da área da superfície lesionada); $87,5 \%=$ Nota 5 (acima de $75 \%$ da área da superfície lesionada). 
Tabela 1. Estimativa do intercepto $(a)$, do coeficiente angular da reta (b) e do coeficiente de determinação $\left(R^{2}\right)$ de equações obtidos a partir da análise de regressão linear simples relacionado a severidade real (determinada eletronicamente com auxílio do programa Assess ${ }^{\circledR}$ ) do oídio (Pseudoidium anacardii) em castanhas de cajueiro (Anacardium occidentale) e a severidade estimada visualmente por vinte avaliadores (dez experientes e dez inexperientes) sem e com o uso da escala diagramática descritiva proposta.

\begin{tabular}{|c|c|c|c|c|c|c|}
\hline \multirow{2}{*}{ Avaliador } & \multicolumn{3}{|c|}{ Sem escala } & \multicolumn{3}{|c|}{ Com escala } \\
\hline & $a$ & $b$ & $R^{2}$ & $a$ & $b$ & $R^{2}$ \\
\hline \multicolumn{7}{|l|}{ Experiente } \\
\hline A & $-0,22$ & $0,89^{*}$ & 0,92 & $-0,83$ & 0,93 & 0,93 \\
\hline B & $6,97^{*}$ & 1,01 & 0,92 & 3,02 & 0,99 & 0,94 \\
\hline $\mathrm{C}$ & $-2,70$ & $1,14^{*}$ & 0,93 & $-1,48$ & $1,10^{*}$ & 0,93 \\
\hline $\mathrm{D}$ & $5,65^{*}$ & 1,08 & 0,90 & $9,71^{*}$ & 0,97 & 0,93 \\
\hline E & $15,82^{*}$ & 0,99 & 0,78 & 1,85 & 1,07 & 0,95 \\
\hline $\mathrm{F}$ & $-1,34$ & 1,01 & 0,94 & $-5,73^{*}$ & 1,08 & 0,95 \\
\hline G & $9,33^{*}$ & 1,05 & 0,93 & 2,71 & 0,97 & 0,96 \\
\hline $\mathrm{H}$ & 0,35 & $1,10^{*}$ & 0,94 & $-3,84^{*}$ & 1,03 & 0,96 \\
\hline I & $21,84^{*}$ & $0,86^{*}$ & 0,86 & $15,68 *$ & $0,85^{*}$ & 0,89 \\
\hline $\mathrm{J}$ & $9,82 *$ & $0,91 *$ & 0,94 & 0,42 & 0,99 & 0,94 \\
\hline Média & 6,55 & 1,00 & 0,91 & 2,15 & 1,00 & 0,94 \\
\hline \multicolumn{7}{|l|}{ Inexperiente } \\
\hline K & 5,99 & 1,03 & 0,78 & 2,71 & $0,88^{*}$ & 0,90 \\
\hline $\mathrm{L}$ & $14,76^{*}$ & 1,04 & 0,86 & $19,40^{*}$ & 0,98 & 0,86 \\
\hline M & 1,40 & $1,15^{*}$ & 0,93 & $8,79 *$ & 1,07 & 0,90 \\
\hline $\mathrm{N}$ & $14,48^{*}$ & 1,09 & 0,78 & $-4,54^{*}$ & $1,20^{*}$ & 0,94 \\
\hline $\mathrm{O}$ & $25,68^{*}$ & $0,88^{*}$ & 0,67 & $12,99 *$ & 0,89 & 0,75 \\
\hline $\mathrm{P}$ & $3,49 *$ & 0,99 & 0,95 & 2,54 & 0,99 & 0,96 \\
\hline Q & 3,73 & $1,10^{*}$ & 0,91 & $-0,01$ & 1,07 & 0,96 \\
\hline $\mathrm{R}$ & $19,09^{*}$ & $0,91^{*}$ & 0,88 & $-0,92$ & 0,93 & 0,93 \\
\hline $\mathrm{S}$ & $7,36^{*}$ & $0,97^{*}$ & 0,79 & 3,23 & 0,93 & 0,92 \\
\hline $\mathrm{T}$ & $17,01^{*}$ & $0,89^{*}$ & 0,88 & $11,28^{*}$ & $0,75^{*}$ & 0,87 \\
\hline Média & 11,30 & 1,01 & 0,84 & 5,55 & 0,97 & 0,90 \\
\hline Média todos & 8,92 & 1,01 & 0,87 & 3,85 & 0,98 & 0,92 \\
\hline
\end{tabular}

*Hipótese de nulidade ( $a=0$ e $b=1)$ rejeitada pelo teste $t(P<0,05)$.

presença, e diminuiu os desvios sistemáticos das estimativas durante a sua validação, contribuindo para a melhora da acurácia das estimativas.

Com o uso da escala, percebe-se que os desvios sistemáticos tenderam a subestimar a severidade do oídio nas castanhas para a maioria dos avaliadores experientes (Figura 2Ac, 2Bc, 2Dc, 2Gc, 2Ic e 2Jc) e inexperientes (Figura $2 \mathrm{Kc}, 2 \mathrm{Lc}, 2 \mathrm{Oc}, 2 \mathrm{Pc}, 2 \mathrm{Rc}, 2 \mathrm{Sc}$ e $2 \mathrm{Tc}$ ), embora de modo muito sutil, com a presença dos valores do coeficiente angular da reta inferiores a unidade, confirmando o que foi relatado anteriormente referente ao coeficiente linear, que indicou tendência de subestimativa por alguns avaliadores. Entretanto, isso não compromete a precisão dos avaliadores (23) e não representa sérios problemas, uma vez que os desvios seguem o mesmo padrão (14) e se aproximam muito de 1 (um), confirmando a acurácia da escala. Além disso, na validação da escala diagramática descritiva, todos os avaliadores (experientes e inexperientes) melhoraram a sua acurácia com o uso da escala (Figura 2 ), pois os valores estimados de severidade ficaram próximos dos valores da severidade real (25).

Houve aumento da precisão das estimativas visuais da severidade do oídio em castanhas de cajueiro com o uso da escala diagramática descritiva proposta, independente da experiência do avaliador, confirmada pelo aumento nos valores dos coeficientes de determinação da regressão (Tabela 1) e pela menor variação dos erros absolutos (Figura 3).

Sem o uso da escala, os coeficientes de determinação obtidos pelos avaliadores variaram de 0,78 a 0,94 para aqueles com experiência, e de 0,67 a 0,95 para os inexperientes, com valores médios das estimativas visuais que explicaram 92 e $87 \%$ da variação na mensuração eletrônica, para avaliadores experientes e inexperientes, respectivamente. Com a utilização da escala, os coefícientes de determinação variaram de 0,89 a 0,96 para avaliadores experientes, e de 0,75 a 0,96 para aqueles inexperientes, com média de 0,94 e 0,91 para avaliadores experientes e inexperientes, respectivamente (Tabela 1).

Utilizando a escala, $80 \%$ dos avaliadores experientes e $70 \%$ daqueles sem experiência melhoraram a precisão das estimativas visuais da severidade do oídio, com valores do coeficiente de determinação mais próximos de 1 (um), demonstrando a eficiência dessa ferramenta em estudos de quantificação de doença $(2,13,20)$.

Os níveis de precisão da severidade do oídio em castanhas de cajueiro com auxílio da escala, para ambos os grupos de avaliadores, com e sem experiência, foram semelhantes aos apresentados em outros trabalhos de validação de escalas $(3,14,27,28,32,38,40)$, com valores dos coeficientes de determinação próximos a 0,95 , considerado desejável por alguns autores $(14,30,38)$. Assim, observa-se que a maioria dos avaliadores apresentou um aumento na acurácia e precisão nas avaliações do oídio em castanhas de cajueiro com o uso da escala diagramática descritiva. Melhoria esta verificada em outros trabalhos, com a utilização de outras escalas diagramáticas $(8,9,11,19,20,22$, 39), demonstrando a importância dessa ferramenta em estudos de quantificação da intensidade de doenças em plantas.

Houve diminuição dos erros absolutos das estimativas da severidade do oídio em castanhas de cajueiro com o auxílio da escala diagramática, independente da experiência do avaliador, quando comparada com a distribuição dos resíduos obtida sem a utilização da escala (Figura 3). Sem o auxílio da escala, a distribuição dos valores dos resíduos das avaliações variou de $-66,68$ a 53,77 (Figura 3Es) para os avaliadores experientes, enquanto que para os avaliadores inexperientes a distribuição dos resíduos variou de $-65,54$ a 60,75 (Figura 3Ks), com média dos erros (em módulo) dos avaliadores experientes igual a 26,12, e 24,65 dos inexperientes.

Quando a escala diagramática descritiva proposta foi utilizada, a distribuição dos resíduos variou de -24,41 (Figura 3Bc) a 29,28 (Figura $3 \mathrm{Cc}$ ) e $-35,87$ a 40,75 (Figura 3Oc) para os avaliadores experientes e inexperientes, respectivamente, reduzindo drasticamente a amplitude dos valores dos resíduos, constatando-se também uma ligeira redução na média dos erros (em módulo), que ficou em 18,32 para os avaliadores experientes e 22,22 para os inexperientes, confirmando a melhora da precisão das estimativas. 


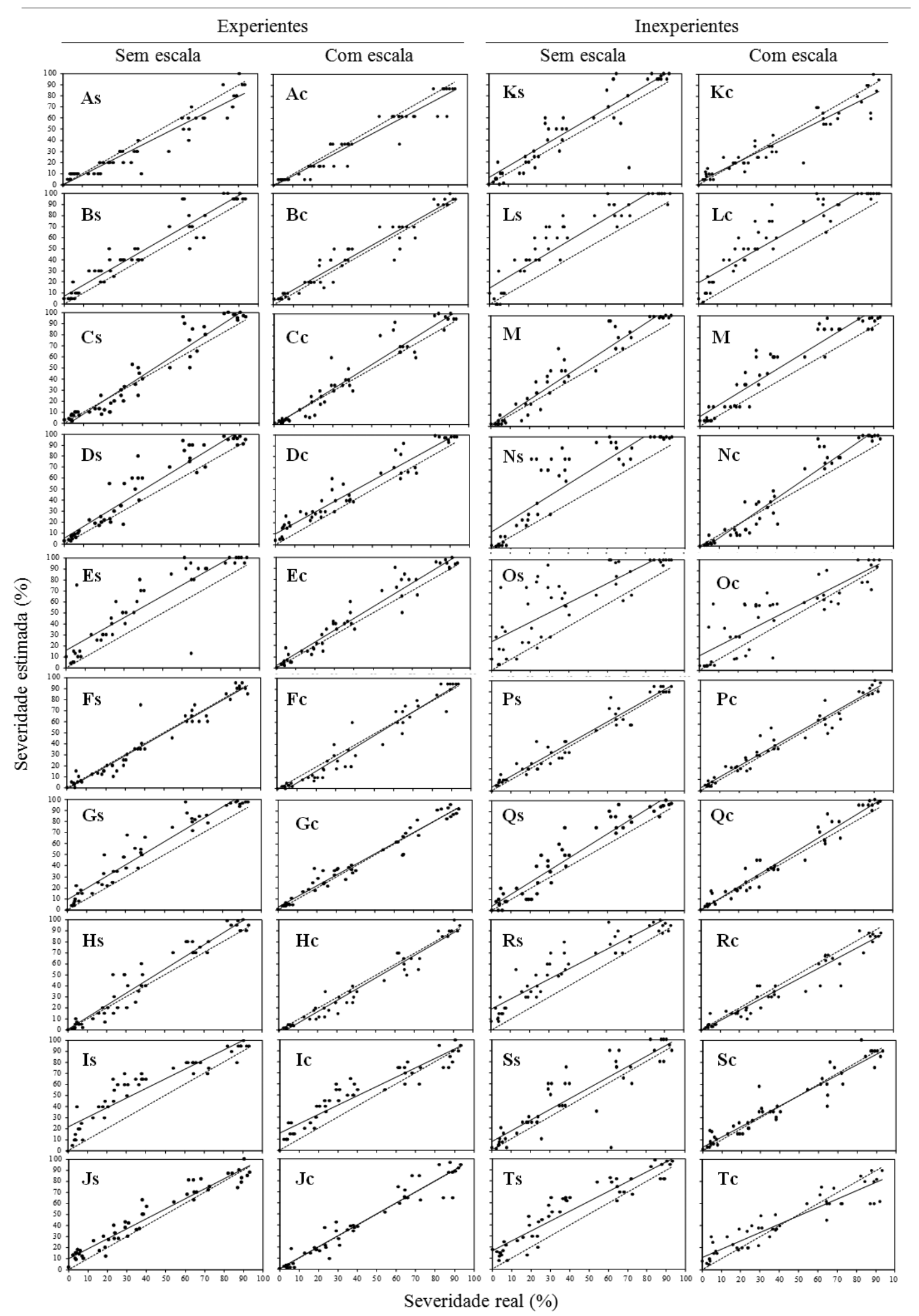

Figura 2. Relação entre severidade real (\%) e severidade estimada (\%) (pontos cheios) para o oídio (Pseudoidium anacardii) em castanhas de cajueiro (Anacardium occidentale) por vinte avaliadores, sendo dez com experiência [sem (As, Bs, Cs, Ds, Es, Fs, Gs, Hs, Is, Js) e com (Ac, Bc, Cc, Dc, Ec, Fc, Gc, Hc, Ic, Jc) o uso da escala proposta] e dez sem experiência [sem (Ks, Ls, Ms, Ns, Os, Ps, Qs, Rs, Ss, Ts) e com (Kc, Lc, Mc, Nc, Oc, Pc, Qc, Rc, Sc, Tc) o uso da escala proposta]. A linha pontilhada, traçada pelo uso dos valores de severidade reais, representa a situação ideal. 

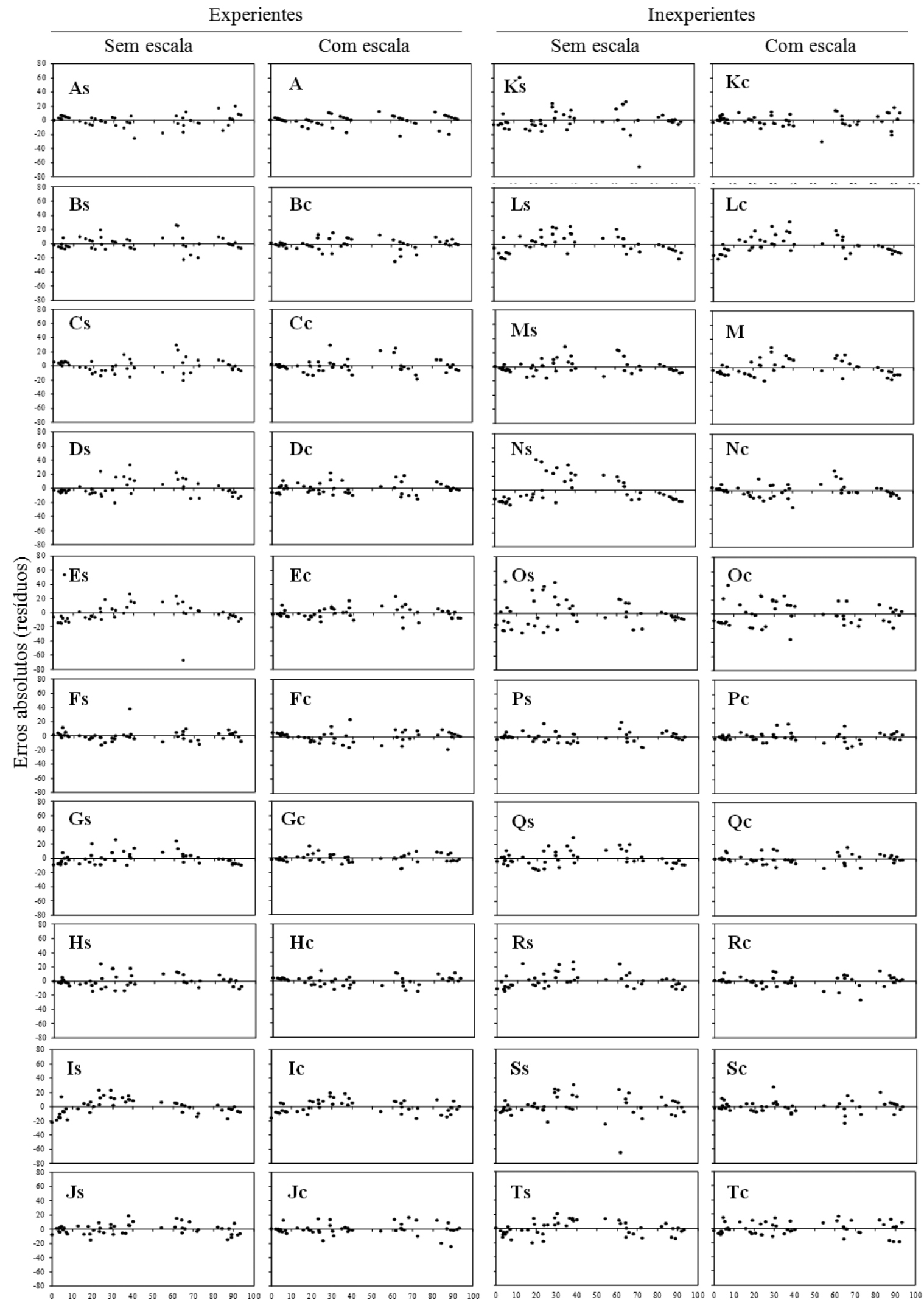

Severidade real (\%)

Figura 3. Erros absolutos (severidade real menos severidade estimada) das estimativas da severidade do para o oídio (Pseudoidium anacardii) em castanhas de cajueiro (Anacardium occidentale) por vinte avaliadores, sendo dez com experiência [sem (As, Bs, Cs, Ds, Es, Fs, Gs, Hs, Is, Js) e com (Ac, Bc, Cc, Dc, Ec, Fc, Gc, Hc, Ic, Jc) o uso da escala proposta] e dez sem experiência [sem (Ks, Ls, Ms, Ns, Os, Ps, Qs, Rs, Ss, Ts) e com (Kc, Lc, Mc, Nc, Oc, Pc, Qc, Rc, Sc, Tc) o uso da escala proposta]. 
Nas avaliações com o uso da escala, os resíduos concentraram-se dentro da faixa de $\pm 10 \%$, valores considerados adequados em estudos de validação de escalas diagramáticas para quantificação da intensidade de doenças em plantas $(3,20,23,24,33)$, e que ainda podem ser minimizados com o treinamento dos avaliadores, visto que mesmo com o uso da escala, ainda existe um certo grau de subjetividade nas estimativas $(2,33)$. A presença de algum tipo de erro absoluto nas mensurações é aceitável e pode ser compensada pela rapidez e padronização resultante do uso da escala $(2,11,20,27)$.

$\mathrm{O}$ uso da escala diagramática descritiva proposta aumentou a reprodutibilidade das estimativas da severidade do oídio em castanhas de cajueiro, obtidas por diferentes avaliadores combinados aos pares (Tabelas 2, 3 e 4).

Sem a utilização da escala, a reprodutibilidade das avaliações foi menor, pois, entre os dez avaliadores experientes, as regressões lineares produziram coeficientes de determinação variando de 0,71 a 0,96 , com valor médio de 0,88 , sendo que em apenas $77,8 \%$ dos casos foi $\geq$ 0,80 e somente $22,2 \%$ foi $\geq 0,90$ (Tabela 2). Entre os inexperientes, as regressões produziram coeficientes de determinação que variaram de 0,62 a 0,93 , com média de 0,81 , em que somente $55,6 \%$ foi $\geq 0,80$ e
$13 \%$ das ocorrências foi $\geq 0,90$ (Tabela 3). Já dentro do grupo formado por experientes e inexperientes, os coeficientes de determinação das regressões lineares variaram de 0,60 a 0,96 , com valor médio de 0,83 , sendo que $57 \% \geq 0,80$ e apenas $24 \%$ foi $\geq 0,90$ (Tabela 4 ).

Com o uso da escala, a reprodutibilidade das avaliações melhorou consideravelmente. Entre os dez avaliadores experientes, as regressões lineares produziram coeficientes de determinação variando de 0,85 a 0,94 , com valor médio de 0,91 , sendo que em $100 \%$ dos casos foi $\geq 0,80$ e em $62 \%$ foi $\geq 0,90$ (Tabela 2). Entre os inexperientes, as regressões produziram coeficientes de determinação que variaram de 0,70 a 0,93 , com média de 0,85 , sendo $73,3 \% \geq 0,80$ e $24 \% \geq 0,90$ (Tabela 3 ). Já dentro do grupo formado por experientes e inexperientes, os coeficientes de determinação das regressões lineares variaram de 0,70 a 0,95 , com valor médio de 0,89 , sendo que em $89 \%$ dos casos foi $\geq 0,80$ e em $44 \%$ $\geq 0,90$ (Tabela 4).

Esses resultados evidenciam o alto nível de reprodutibilidade das estimativas obtidas por diferentes grupos de avaliadores na avaliação do oídio em castanhas de cajueiro, assemelhando-se ao constatado na validação de escalas diagramáticas por outros autores em diferentes patossistemas $(3,24,28,30)$. O aumento da reprodutibilidade com o

Tabela 2. Estimativa do coeficiente de determinação $\left(R^{2}\right)$ de equações de regressão linear simples relacionando as estimativas do oídio $(P s e u d o i d i u m ~$ anacardii) em castanhas de cajueiro (Anacardium occidentale) entre avaliadores experientes sem e com o auxílio da escala diagramática descritiva proposta.

\begin{tabular}{|c|c|c|c|c|c|c|c|c|c|c|c|c|c|c|c|c|c|c|}
\hline \multirow{2}{*}{ Avaliador } & \multicolumn{9}{|c|}{ Sem escala } & \multicolumn{9}{|c|}{ Com escala } \\
\hline & $\mathrm{B}$ & $\mathrm{C}$ & $\mathrm{D}$ & E & $\mathrm{F}$ & G & $\mathrm{H}$ & I & $\mathrm{J}$ & $\mathrm{B}$ & $\mathrm{C}$ & $\mathrm{D}$ & E & $\mathrm{F}$ & G & $\mathrm{H}$ & I & $\mathrm{J}$ \\
\hline A & 0,85 & 0,89 & 0,79 & 0,71 & 0,89 & 0,81 & 0,87 & 0,75 & 0,86 & 0,89 & 0,90 & 0,91 & 0,92 & 0,89 & 0,90 & 0,90 & 0,86 & 0,93 \\
\hline B & & 0,89 & 0,89 & 0,75 & 0,87 & 0,89 & 0,93 & 0,87 & 0,90 & & 0,90 & 0,89 & 0,93 & 0,89 & 0,92 & 0,91 & 0,88 & 0,91 \\
\hline $\mathrm{C}$ & & & 0,89 & 0,79 & 0,92 & 0,91 & 0,89 & 0,77 & 0,89 & & & 0,93 & 0,94 & 0,88 & 0,92 & 0,93 & 0,88 & 0,90 \\
\hline D & & & & 0,80 & 0,89 & 0,91 & 0,92 & 0,86 & 0,92 & & & & 0,92 & 0,91 & 0,92 & 0,92 & 0,87 & 0,89 \\
\hline E & & & & & 0,74 & 0,79 & 0,79 & 0,77 & 0,81 & & & & & 0,89 & 0,93 & 0,92 & 0,89 & 0,92 \\
\hline F & & & & & & 0,89 & 0,88 & 0,81 & 0,89 & & & & & & 0,92 & 0,92 & 0,85 & 0,89 \\
\hline G & & & & & & & 0,91 & 0,88 & 0,90 & & & & & & & 0,93 & 0,85 & 0,93 \\
\hline $\mathrm{H}$ & & & & & & & & 0,87 & 0,96 & & & & & & & & 0,86 & 0,91 \\
\hline I & & & & & & & & & 0,87 & & & & & & & & & 0,86 \\
\hline
\end{tabular}

Tabela 3. Estimativa do coeficiente de determinação $\left(R^{2}\right)$ de equações de regressão linear simples relacionando as estimativas do oídio $(P s e u d o i d i u m ~$ anacardii) em castanhas de cajueiro (Anacardium occidentale) entre avaliadores inexperientes sem e com o auxílio da escala diagramática descritiva proposta.

\begin{tabular}{|c|c|c|c|c|c|c|c|c|c|c|c|c|c|c|c|c|c|c|}
\hline \multirow{2}{*}{ Avaliador } & \multicolumn{9}{|c|}{ Sem escala } & \multicolumn{9}{|c|}{ Com escala } \\
\hline & $\mathrm{L}$ & $\mathrm{M}$ & $\mathrm{N}$ & $\mathrm{O}$ & $\mathrm{P}$ & Q & $\mathrm{R}$ & $\mathrm{S}$ & $\mathrm{T}$ & $\mathrm{L}$ & $\mathrm{M}$ & $\mathrm{N}$ & $\mathrm{O}$ & $\mathrm{P}$ & Q & $\mathrm{R}$ & $\mathrm{S}$ & $\mathrm{T}$ \\
\hline K & 0,81 & 0,81 & 0,67 & 0,62 & 0,84 & 0,78 & 0,82 & 0,76 & 0,76 & 0,80 & 0,84 & 0,88 & 0,73 & 0,90 & 0,91 & 0,89 & 0,87 & 0,82 \\
\hline $\mathrm{L}$ & & 0,90 & 0,87 & 0,73 & 0,87 & 0,85 & 0,93 & 0,77 & 0,87 & & 0,87 & 0,83 & 0,71 & 0,88 & 0,82 & 0,78 & 0,76 & 0,79 \\
\hline M & & & 0,81 & 0,77 & 0,93 & 0,92 & 0,91 & 0,78 & 0,87 & & & 0,88 & 0,79 & 0,88 & 0,89 & 0,85 & 0,85 & 0,81 \\
\hline $\mathrm{N}$ & & & & 0,67 & 0,78 & 0,81 & 0,82 & 0,65 & 0,81 & & & & 0,72 & 0,91 & 0,93 & 0,90 & 0,90 & 0,89 \\
\hline $\mathrm{O}$ & & & & & 0,71 & 0,70 & 0,71 & 0,62 & 0,66 & & & & & 0,77 & 0,77 & 0,73 & 0,77 & 0,70 \\
\hline $\mathrm{P}$ & & & & & & 0,90 & 0,89 & 0,75 & 0,85 & & & & & & 0,93 & 0,91 & 0,87 & 0,84 \\
\hline Q & & & & & & & 0,89 & 0,73 & 0,88 & & & & & & & 0,92 & 0,93 & 0,85 \\
\hline $\mathrm{R}$ & & & & & & & & 0,80 & 0,89 & & & & & & & & 0,91 & 0,82 \\
\hline $\mathrm{S}$ & & & & & & & & & 0,77 & & & & & & & & & 0,86 \\
\hline
\end{tabular}


Tabela 4. Estimativa do coeficiente de determinação $\left(R^{2}\right)$ de equações de regressão linear simples relacionando as estimativas do oídio $(P s e u d o i d i u m ~$ anacardii) em castanhas de cajueiro (Anacardium occidentale) entre avaliadores experientes (A, B, C, D, E, F, G, H, I, J) e inexperientes (K, L, $\mathrm{M}, \mathrm{N}, \mathrm{O}, \mathrm{P}, \mathrm{Q}, \mathrm{R}, \mathrm{S}, \mathrm{T})$ sem e com o auxílio da escala diagramática descritiva proposta.

\begin{tabular}{|c|c|c|c|c|c|c|c|c|c|c|c|c|c|c|c|c|c|c|c|c|}
\hline \multirow{2}{*}{$\begin{array}{l}\text { Avaliador } \\
\text { experiente }\end{array}$} & \multicolumn{10}{|c|}{ Avaliador inexperiente sem escala } & \multicolumn{10}{|c|}{ Avaliador inexperiente com escala } \\
\hline & $\mathrm{K}$ & $\mathrm{L}$ & $\mathrm{M}$ & $\mathrm{N}$ & $\mathrm{O}$ & $\mathrm{P}$ & Q & $\mathrm{R}$ & $\mathrm{S}$ & $\mathrm{T}$ & $\mathrm{K}$ & $\mathrm{L}$ & M & $\mathrm{N}$ & $\mathrm{O}$ & $\mathrm{P}$ & Q & $\mathrm{R}$ & $\mathrm{S}$ & $\mathrm{T}$ \\
\hline A & 0,73 & 0,74 & 0,86 & 0,64 & 0,63 & 0,90 & 0,83 & 0,79 & 0,77 & 0,79 & 0,89 & 0,79 & 0,88 & 0,90 & 0,78 & 0,90 & 0,91 & 0,90 & 0,90 & 0,83 \\
\hline B & 0,78 & 0,88 & 0,93 & 0,79 & 0,71 & 0,95 & 0,89 & 0,89 & 0,71 & 0,84 & 0,84 & 0,82 & 0,88 & 0,88 & 0,76 & 0,92 & 0,94 & 0,91 & 0,90 & 0,81 \\
\hline C & 0,76 & 0,79 & 0,93 & 0,70 & 0,68 & 0,92 & 0,89 & 0,84 & 0,73 & 0,85 & 0,86 & 0,87 & 0,89 & 0,93 & 0,74 & 0,92 & 0,93 & 0,87 & 0,89 & 0,86 \\
\hline D & 0,80 & 0,89 & 0,95 & 0,85 & 0,72 & 0,91 & 0,94 & 0,92 & 0,75 & 0,87 & 0,87 & 0,83 & 0,88 & 0,90 & 0,77 & 0,91 & 0,94 & 0,90 & 0,92 & 0,84 \\
\hline E & 0,60 & 0,75 & 0,78 & 0,73 & 0,60 & 0,76 & 0,79 & 0,79 & 0,62 & 0,81 & 0,88 & 0,88 & 0,91 & 0,95 & 0,74 & 0,91 & 0,94 & 0,91 & 0,93 & 0,89 \\
\hline $\mathrm{F}$ & 0,79 & 0,83 & 0,89 & 0,74 & 0,64 & 0,91 & 0,87 & 0,86 & 0,78 & 0,83 & 0,89 & 0,76 & 0,87 & 0,90 & 0,71 & 0,91 & 0,93 & 0,90 & 0,90 & 0,82 \\
\hline G & 0,79 & 0,91 & 0,92 & 0,81 & 0,72 & 0,91 & 0,89 & 0,90 & 0,79 & 0,90 & 0,87 & 0,85 & 0,87 & 0,91 & 0,75 & 0,94 & 0,95 & 0,90 & 0,92 & 0,85 \\
\hline $\mathrm{H}$ & 0,78 & 0,89 & 0,93 & 0,82 & 0,75 & 0,96 & 0,91 & 0,90 & 0,78 & 0,87 & 0,91 & 0,83 & 0,85 & 0,93 & 0,70 & 0,95 & 0,94 & 0,90 & 0,90 & 0,86 \\
\hline I & 0,75 & 0,92 & 0,86 & 0,88 & 0,77 & 0,85 & 0,87 & 0,88 & 0,77 & 0,85 & 0,85 & 0,87 & 0,91 & 0,85 & 0,80 & 0,88 & 0,89 & 0,85 & 0,87 & 0,83 \\
\hline $\mathrm{J}$ & 0,77 & 0,88 & 0,93 & 0,80 & 0,71 & 0,93 & 0,92 & 0,90 & 0,76 & 0,87 & 0,89 & 0,83 & 0,89 & 0,92 & 0,74 & 0,91 & 0,94 & 0,92 & 0,88 & 0,84 \\
\hline
\end{tabular}

uso da escala diagramática, obtidas por diferentes avaliadores, mostra que, do ponto de vista prático, experimentos distintos, conduzidos por vários pesquisadores, podem ser comparados $(2,26,27)$, desde que se utilizem a escala para a avaliação do oídio em castanhas de cajueiro, uma vez que os dados aqui estimados por diversos avaliadores estiveram próximos a uma concordância perfeita, indicando alta precisão $(30,33)$.

A escala mostrou ser de fácil utilização e pode ser usada, inclusive, por pessoas sem experiência na quantificação de doenças de plantas, comprovando ser esta uma ferramenta útil para avaliações de severidade desta fitomoléstia mesmo quando utilizada por pessoas sem nenhum treinamento ou conhecimento prévio na quantificação de doenças.

Uma boa escala é aquela a qual diferentes avaliadores, ao utilizála para a avaliação da intensidade de doença do mesmo material, estimam os mesmos valores de severidade $(24,33)$, e os resultados podem ser comparados entre si, ficando então demonstrando que a reprodutibilidade das avaliações do oídio em castanhas de cajueiro foi garantida com o uso da escala diagramática descritiva desenvolvida.

Com os resultados obtidos é possível estabelecer um sistema padronizado de quantificação da intensidade de doença, visando orientar a avaliação da severidade do ataque do oídio em plantas de cajueiro. Quantificar doenças trata-se de uma atividade de grande responsabilidade, pois, caso haja ineficiência do sistema, o custo oriundos do seu uso pode superar os benefícios advindos de sua utilização (33), visto que estimativas visuais imprecisas levam a erros que alteram conclusões de ensaios e comprometem resultados (20, 35). Entretanto, a padronização do sistema de quantificação do oídio é extremamente necessária, pois a uniformização da metodologia de avaliação dessa fitomoléstia permite comparações entre os resultados obtidos por pesquisadores em diferentes instituições, localizadas em diferentes regiões e em períodos distintos (14).

A escala diagramática descritiva proposta melhora os níveis de acurácia, precisão e reprodutibilidade das mensurações da severidade do oídio em castanhas de cajueiro independente da experiência do avaliador. Esta escala poderá ser adotada em estudos do oídio do cajueiro, sendo uma excelente ferramenta de auxílio para reduzir e minimizar possíveis erros de estimativa da doença em condições de campo, diminuindo a subjetividade das estimativas e propiciando informações precisas sobre a intensidade da doença nesse importante patossistema. Esta ferramenta poderá ser aplicada a uma ampla gama de situações como levantamentos de campo, estudos epidemiológicos de progresso e disseminação do oídio, resistência de plantas de cajueiro, como também em estudos de medidas de controle da doença e poderá ser usada por estudantes, técnicos e pesquisadores no Brasil e em outros países onde a doença ocorre.

\section{AGRADECIMENTOS}

À Coordenação de Aperfeiçoamento de Pessoal de Nível Superior pela bolsa de doutorado concedida ao primeiro autor.

\section{REFERÊNCIAS}

1. Amorim, L; Bergamin Filho, A. Fenologia, patometria e quantificação de danos. In: Amorim, L.; Rezende, J.A.M.; Bergamin Filho, A. (Ed.). Manual de fitopatologia. Piracicaba: Agronômica Ceres, 2011. p.517-542.

2. Barbosa, M.A.G.; Michereff, S.J.; Mora-Aguilera, G. Elaboração e validação de escala diagramática para avaliação da severidade da ferrugem branca do crisântemo. Summa Phytopathologica, Botucatu, v.32, n.1, p.57-62, 2006.

3. Barguil, B.M.; Albert, I.C.L.; Michereff, S.J.; Oliveira, S.M.A. Escala diagramática para avaliação da severidade da antracnose em bastão do imperador. Ciência Rural, Santa Maria, v.38, n.3, p.807-810, 2008.

4. Bedendo, I.P. Oídios. In: Amorim, L.; Rezende, J.A.M.; Bergamin Filho, A. (Ed.). Manual de fitopatologia. Piracicaba: Agronômica Ceres, 2011. p.473-477.

5. Braun, U.; Cook, R.T.A. Taxonomic Manual of the Erysiphales (Powdery Mildews). Saint Paul: American Phytopathological Society, 2012. 707p.

6. Cardoso, J.E.; Martins, M.V.V.; Lima, J.S.; Viana, F.M.P.; Silva, L.G.C. Controle químico do oídio do cajueiro. Fortaleza: Embrapa Agroindústria Tropical, 2012. 4p. (Comunicado Técnico, 196).

7. Cardoso, J.E.; Santos, A.A.; Freire, F.C.O.; Viana, F.M.P.; Vidal, J.C.; Oliveira, J.N.; Uchoa, C.N. Monitoramento de doenças na cultura do cajueiro. Fortaleza: Embrapa Agroindústria Tropical, 2006. 24p. (Documentos, 47).

8. Citadin, I.; Assmann, A.P.; Mazaro, S.M.; Gouvêa, A.; Danner, M.A.; Malagi, G. Escala diagramática para avaliação da severidade de bacteriose em pessegueiro. Revista Brasileira de Fruticultura, Jaboticabal, v.30, n.2, p.327-330, 2008.

9. Damasceno, V.F.F.; Furtado, E.L.; Ferreira Filho, P.J. Comparação de dois métodos de elaboração e validação de escala diagramática para a quantificação da severidade da mancha de Cylindrocladium em eucalipto. Summa Phytopathologica, Botucatu, v.40, n.3, p.248-255, 2014. 
10. FAO. Production. Roma, 2016. Disponível em: <http://faostat3.fao.org/ faostat-gateway/go/to/download/Q/QC/E>. Acesso em: 20 dez. 2016.

11. Fischer, I.H. Alves, S.A.M.; Almeida, A.M.; Arruda, M.C.; Bertani, R.M.A.; Garcia, M.J.M. Elaboration and validation of diagrammatic scale to evaluate anthracnose severity in yellow passion fruits. Summa Phytopathologica, Botucatu, v.35, n.3, p.226-228, 2009.

12. Freire, F.C.O.; Cardoso, J.E.; Santos, A.A.; Viana, F.M.P. Diseases of cashew nut plants (Anacardium occidentale L.) in Brazil. Crop Protection, Kent, v.21, n.6, p.489-494, 2002.

13. Godoy, C.V.; Koga, L.J.; Canteri, M.G. Diagrammatic scale for assessment of soybean rust severity. Fitopatologia Brasileira, Brasília, DF, v.31, n.1, p.63-68, 2006.

14. Gomes, A.M.A.; Michereff, S.J.; Mariano, R.L.R. Development and validation of a diagramatic key for Cercospora leaf spot of lettuce. Summa Phytopathologica, Botucatu, v.30, n.1, p.38-42, 2004.

15. Honorato, T.B.; Lima, J.S.; Cardoso, J.E. Caracterização morfofisiológica e sensibilidade de Pseudoidium anacardii a fungicidas. Fortaleza: Embrapa Agroindústria Tropical, 2016. 23p. (Boletim de Pesquisa e Desenvolvimento, 119)

16. Horsfall, J.G.; Cowling, E.B. Pathometry: the measurement of plant disease. In: Horsfall, J.G.; Cowling, E.B. (Ed.). Plant disease: an advanced treatise - how disease develops in populations. New York: Academic Press, 1978. v.2, p.119-136.

17. Instituto Brasileiro de Geografia e Estatística. Produção agrícola municipal. Rio de Janeiro, 2016. Disponível em: <http://www.sidra.ibge.gov. $\mathrm{br} / \mathrm{bda} / \mathrm{tabela} / \mathrm{listab} 11 . \mathrm{asp} ? \mathrm{c}=1613 \& \mathrm{n}=0 \& \mathrm{u}=0 \& \mathrm{z}=\mathrm{p} \& \mathrm{o}=18 \& \mathrm{i}=\mathrm{P}>$. Acesso em: 20 dez. 2016.

18. Lamari, L. Assess: Image analysis software for plant disease quantification. Sant Paul: APS Press, 2002. 1 CD-ROM

19. Lazaroto, A.; Santos, I.; Konflanz, V.M.; Malagi, G.; Camochena, R.C. Escala diagramática para avaliação de severidade da helmintosporiose comum em milho. Ciência Rural, Santa Maria, v.42, n.12, p.2288-2294, 2012.

20. Lenz, G.; Balardin, R.S.; Corte, G.D.; Marques, L.N.; Debona, D. Escala diagramática para avaliação de severidade de mancha-parda em arroz. Ciência Rural, Santa Maria, v.40, n.4, p.752-758, 2010.

21. Lima, J.S. Epidemiologia quantitativa do oídio do cajueiro no clone BRS 189. 2017. 82f. Tese (Doutorado em Agronomia/Fitotecnia)-Centro de Ciências Agrárias, Universidade Federal do Ceará, Fortaleza.

22. Maciel, J.L.N.; Danelli, A.L.D.; Boretto, C.; Forcelini, C.A. Diagrammatic scale for assessment of blast on wheat spikes. Summa Phytopathologica, Botucatu, v.39, n.3, p.162-166, 2013.

23. Malagi, G.; Santos, I.; Camochena, R.C.; Moccellin, R. Elaboração e validação da escala diagramática para avaliação da mancha branca do milho. Revista Ciência Agronômica, Fortaleza, v.42, n.3, p.797-804, 2011.

24. Martins, M.C.; Guerzoni, R.A.; Câmara, G.M.S.; Mattiazzi, P.; Lourenço, S.A.; Amorim, L. Escala diagramática para a quantificação do complexo de doenças foliares de final de ciclo em soja. Fitopatologia Brasileira, Brasília, DF, v.29, n.2, p.179-184, 2004.

25. Mazaro, S.M.; Golvea, A.; De Mio L.L.M.; Deschamps, C.; Biasi, L.A.;
Citadin, I. Escala diagramática para avaliação da severidade da manchade-micosferela em morangueiro. Ciência Rural, Santa Maria, v.36, n.2, p.648-652, 2006

26. Michereff, S.J.; Andrade, D.E.G.T.; Noronha, M.A. Elaboração e validação de escala diagramática para avaliação da severidade do carvão da folha do caupi. Summa Phytopathologica, Botucatu, v.32, n.1, p.51-56, 2006.

27. Michereff, S.J.; Noronha, M.A.; Andrade, D.E.G.T.; Oliveira, E.P.; Xavier Filha, M.S.; Moreira, P.A.A. Development and validation of a diagrammatic key for Cercospora leaf spot of sweet pepper. Summa Phytopathologica, Botucatu, v.32, n.3, p.260-266, 2006

28. Michereff, S.J.; Noronha, M.A.; Lima, G.S.A.; Albert, I.CL.; Melo, E.A Gusmão, L.O. Diagrammatic scale to assess downy mildew severity in melon. Horticultura Brasileira, Vitória da Conquista, v.27, n.1, p.76-79, 2009

29. Mizubuti, E.S.G.; Maffia, L.A. Introdução à Fitopatologia. Viçosa: Universidade Federal de Viçosa, 2006. 190p. (Cadernos Didáticos, 115).

30. Nascimento, A.R.P.; Michereff, S.J.; Mariano, R.L.R.; Gomes, A.M.A. Elaboração e validação de escala diagramática para cancro bacteriano da videira. Summa Phytopathologica, Botucatu, v.31, n.1, p.59-64, 2005

31. Nita, M.; Ellis, M.A.; Madden, L.V. Effects of temperature, wetness duration and leaflet age on infection of strawberry foliage by Phomopsis obscurans. Plant Disease, Saint Paul, v.87, n.1 p.579-590, 2003.

32. Nunes, C.C.; Alves, S.A.M. Elaboração e validação de escala diagramática para quantificação da severidade de entomosporiose em folhas de pereira. Summa Phytopathologica, Botucatu, v.38, n.3, p.239-244, 2012.

33. Nutter Jr., F.W.; Schultz, P.M. Improving the accuracy and precision of disease assessments: selection of methods and use of computer- aided training programs. Canadian Journal of Plant Pathology, Ottawa, v.17, n.1, p.174-184, 1995.

34. Oliveira, V.H. Cajucultura. Revista Brasileira de Fruticultura, Jaboticabal, v.30, n.1, p.1-3, 2008

35. Parker, S.R.; Shaw, M.W.; Royle, D.J. The reliability of visual estimates of disease severity on cereal leaves. Plant Pathology, London, v.44, n.5, p.856-864, 1995 .

36. Pinto, O.R.O.; Muniz, C.R.; Cardoso, J.E.; Oliveira, F.S.A.; Lima, J.S. Morphological analyses of Pseudoidium anacardii infecting Brazilian cashew plants. Summa Phytopathologica, Botucatu, v.42, n.3, p.257-260, 2016.

37. Renaud, M.S.A.; Amorim, L.; Lourenço, S.A.; Spósito, M.B. Escala diagramática para avaliação da mancha marrom de alternaria de citros. Summa Phytopathologica, Botucatu, v.34, n.3, p.270-271, 2008

38. Sachs, P.J.D.; Neves, C.S.V.J.; Canteri, M.G.; Sachs, L.G. Escala diagramática para avaliação da severidade da mancha branca em milho. Summa Phytopathologica, Botucatu, DF, v.37, n.4, p.202-204, 2011

39. Spósito, M.B.; Amorim, L.; Belasque Junior, J.; Bassanezi, R.B.; Aquino, R. Elaboração e validação de escala diagramática para avaliação da severidade da mancha preta em frutos cítricos. Fitopatologia Brasileira, Brasília, DF, v.29, n.1, p.81-85, 2004.

40. Vivas, M.; Terra, C.E.P.S.; Silveira, S.F.; Fontes, R.V.; Pereira, M.G. Diagrammatic scale for assessing severity of back-spot in papaya fruit. Summa Phytopathologica, Botucatu, v.36, n.2, p.161-163, 2010. 